\title{
Revisión de literatura de la teoría del comportamiento planificado en la decisión de compra de productos orgánicos
}

\author{
Literature review the theory of planned behavior in the decision \\ to buy organic products
}

DOI: $10.22458 /$ rna.v12i1.3178

\begin{abstract}
Mag. Daniel Yiwady Ordoñez Abril ${ }^{1}$ Mag. Jaime Hernán Calderón Sotero ${ }^{1}$ Mag. Lina Marcela Padilla Delgado²

${ }^{1}$ LUMEN GENTIUM Catholic University Foundation, Faculty of Business Sciences, Cali, Colombia; dyordoneza@unicatolica.edu.co, https://orcid.org/0000-0002-5598-0662; jhcalderon@unicatolica.edu.co, https://orcid.org/0000-0002-8200-478X

${ }^{2}$ LUMEN GENTIUM Catholic University Foundation, Faculty of Business Sciences, Universidad San Buenaventura, Cali, Colombia; linamarce942@gmail.com, https://orcid.org/0000-0001-9999-097X
\end{abstract}

\section{RESUMEN}

Actualmente, el consumo responsable hacia el medio ambiente y la salud modifica la intención de compra, que es analizada ampliamente en la teoría del comportamiento planificado (TPB). Este artículo revisa bibliográficamente la aplicación de la TPB y para ello sigue la metodología de consulta de artículos científicos publicados y enfocados en determinar la intención de comprar productos orgánicos. Se encuentran diversos estudios centrados en analizar la preocupación por el ambiente, el comportamiento hacia la sostenibilidad ambiental, los hábitos saludables, los beneficios ambientales de los productos orgánicos y la confianza del consumidor frente a este tipo de productos. Se evidencia que la TPB permite determinar intenciones y comportamientos de compra, los cuales pueden ser influenciados por características culturales y sociales de los individuos, el conocimiento y la preocupación por el medio ambiente.

Clasificación JEL: (Q5-M31-M30)

\begin{abstract}
Nowadays responsible consumption towards the environment and health, modify the intention to buy, which is widely analyzed in planned behavior theory (PBT). . This article bibliographically reviews the application of PBT, by following the consultation methodology of published scientific articles focused on determining the intention to purchase organic products. There are several studies focused on analyzing environmental concern, the behavior towards environmental sustainability, healthy habits, the environmental benefits of organic products and the consumer's confidence towards these types of products. There is evidence that PBT allows to determine intentions and behaviors to purchase, which can be influenced by cultural and social characteristics of individuals, knowledge and environmental concern.

JEL Classification Codes: (Q5-M31-M30)
\end{abstract}

\section{RÉSUMÉ}

Actuellement, la consommation responsable envers l'environnement et la santé modifie l'intention d'achat, qui est analysée amplement dans la théorie du comportement planifié (TPB en anglais). Cet article fait une révision bibliographique de l'application de la TPB et pour ce faire il suit la méthodologie de consultation des articles scientifiques publiés et centrés sur la question de déterminer l'intention d'achat de produits organiques. Il y a plusieurs études centrées sur l'analyse de la préoccupation pour l'environnement, le comportement envers la durabilité environnementale, les habitudes saines, les avantages environnementaux des produits organiques et la confiance du consommateur dans ce type de produits. Il est prouvé que la TPB permet de déterminer les intentions et les comportements d'achat, qui peuvent être influencés par des caractéristiques culturelles et sociales des individus, la connaissance et la préoccupation pour l'environnement. Classification JEL : (Q5-M31-M30)

\section{RESUMO}

Atualmente, o consumo responsável com o meio ambiente e a saúde modifica a intenção de compra, amplamente analisada na teoria do comportamento planejado (TPB). Este artigo faz uma revisão bibliográfica da aplicação do TPB e para isso segue a metodologia de consulta a artigos científicos publicados e focados em determinar a intenção de compra de produtos orgânicos. Encontram-se diversos estudos centrados em analisar a preocupação com o meio ambiente, o comportamento em relação à sustentabilidade ambiental, os hábitos saudáveis, os benefícios ambientais dos produtos orgânicos e a confiança do consumidor perante este tipo de produtos. Evidencia-se que o TPB permite determinar intenções e comportamentos de compra, os quais podem ser influenciados por características culturais e sociais dos indivíduos o conhecimento e a preocupação com o meio ambiente. Classificação JEL: (Q5-M31-M30)

\section{PALABRAS CLAVE: \\ COMPORTAMIENTO DE COMPRA, INTENCIÓN DE COMPRA, ACTITUD, NORMAS SUBJETIVAS, CONTROLES PERCIBIDOS.}
KEYWORDS:
BEHAVIOR TO PURCHASE, INTENTION TO PURCHASE, ATTITUDE, SUBJECTIVE NORMS, PERCEIVED CONTROL.

MOTS-CLÉS

COMPORTEMENT

D'ACHAT, INTENTION

D'ACHAT, ATITUDE,

NORMES SUBJECTIVES,

CONTRÔLES PERÇUS.
PALAVRAS-CHAVE:

COMPORTAMENTO DE COMPRA, INTENÇÃO DE COMPRA, ATITUDE, NORMAS SUBJETIVAS, CONTROLES PERCEBIDOS. 


\section{INTRODUCCIÓN}

Los estudios sobre el marketing ecológico tienen sus primeras aproximaciones a principios de la década de los setenta, con autores como Kassarjian (1971), Fisk (1974) y Henion y Kinnear (1976), quienes manifestaron preocupaciones por su entorno y las repercusiones de sus decisiones de compra como consumidores verdes (Newholm y Shaw, 2007). El interés resurgió en la década de los noventa con autores como Prothero (1990), Ottman (1992), Peattie y Ratnayaka (1992), Coddington (1993), Fuller y Butler (2015), Polanski y Anderson (2006), Van-Dam y Apeldoorn (1996) y Fuller (1999). Estos se enfocaron en los alcances mercadológicos de los productos, la legislación ambiental y las expectativas de los consumidores con la protección del medio ambiente, donde el consumo verde se definió como aquel que evita:

Productos que ponen en riesgo la salud del consumidor o de otro; causan daño significativo al medio ambiente durante la manufactura, su uso o desperdicio; consumen una cantidad desproporcionada de energía; causan un desperdicio innecesario; usan materiales derivados de especies o ambientes amenazados; así como aquellos que implican un maltrato innecesario de animales o que de manera adversa afectan a otros países. (Elkington y Hailes, 1989, p. 235)

En ese contexto, la definición incorporó enfoques éticos que implican las decisiones de consumo, lo cual da lugar al concepto de consumidor ético; este se interesa no solo en la dimensión medioambiental de sus decisiones, sino también en aspectos morales y éticos (Shaw y Shiu, 2002). Este tipo de consumidor cuestiona las malas conductas de compras, resiste a los esfuerzos del marketing y prefiere un estilo de vida más simple y saludable (Newholm y Shaw, 2007). Es decir, los consumidores deben ser entendidos como agentes de los procesos de consumo, que tienen una responsabilidad en la toma de decisiones con respecto a los productos que consumen.

Más adelante, estas acepciones, en cierta manera, son absorbidas por un concepto más amplio: el consumidor socialmente responsable, quien, además de preocuparse por el medio ambiente y la ética, es consciente de que sus actos de consumo repercuten en su entorno. Por lo tanto, indaga en las acciones de responsabilidad social de las empresas, así como en los procesos productivos y las prácticas, busca información adicional a la proporcionada en los bienes y servicios (Mohr et al., 2001), es consciente de que sus decisiones tienen repercusiones en la sociedad y, por ende, reconoce su rol como agente de cambio social (Wang y Strong, 1996).

Así, los estudios de los últimos cincuenta años han evidenciado que los consumidores compran por impulso, por necesidad y para sentir alivio, donde hay "muchas variables para estudiar que van más allá del precio: factores culturales, sociales, personales y psicológicos” (Raiteri 2015, p. 15). De ahí que descifrar el comportamiento del comprador sea, según Galindo (2001), "un reto difícil de lograr, pero indispensable desafiar para llegar a entender el complejo conjunto de estímulos y emociones que lo influyen a la hora de tomar una decisión de compra” (p. 4), lo cual posibilita la emergencia de teorías del comportamiento aplicadas en el marketing y que pretenden hacer un llamado a la responsabilidad y al empoderamiento que tiene el consumidor con el medio ambiente e incentivar a las empresas a pensar en modelos de negocios y estrategias que favorezcan sus utilidades, satisfagan las necesidades de los consumidores y se responsabilicen de prácticas ambientales sostenibles (Sánchez-Villegas, 2014). Estas, en gran medida, según Pita-Morales (2016), han sido desarrolladas por "una serie de instancias, normas y leyes que promueven un cambio de actitud y de posturas de los ciudadanos frente al cuidado del planeta y sus recursos" (p. 123).

Ahora bien, las publicaciones recientes exponen modelos más holísticos, los cuales integran variables psicosociales y perspectivas más funcionales, y extienden teorías ampliamente estudiadas, como la teoría de acción razonada y la teoría del comportamiento planificado; estas han sido aplicadas con éxito en estudios de intenciones y comportamiento del consumidor con respecto a los alimentos orgánicos (ver, por ejemplo, Batista Ferraz et al., 2017; Chen y Lobo, 2012; Choi y Johnson, 2019; Nuttavuthisit y Thøgersen, 2017; Al-Mamun et al., 2018; Zheng y Chi, 2015; Qi y Ploeger, 2019; Oliveira-Brochado et al., 2015; Singh y Verma, 2017; entre otros).

Por lo tanto, emerge el cuestionamiento: ¿cómo se puede caracterizar la literatura sobre la teoría del comportamiento planificado en términos de teorías, métodos y hallazgos sobre la decisión de compra de productos ecológicos? Este artículo presenta de manera panorámica un repaso de la literatura sobre la teoría del comportamiento planificado en los estudios de compra de productos orgánicos. Para esto, se utilizan, como fuentes de datos, publicaciones de revistas indexadas según los índices SJR-Scopus de niveles Q1, Q2, Q3 y Q4, y se considera el campo del marketing ecológico y verde. 
El estudio se estructura a partir de cuatro secciones adicionales a la introducción. La primera describe el método de investigación empleado; la segunda sección presenta conceptos básicos de la teoría del comportamiento planificado y su incidencia en la intención de compra; la siguiente sección presenta el análisis de los resultados y las discusiones; por último, en las consideraciones finales, se proponen, a la luz de los resultados encontrados, posibilidades de investigación que envuelvan el asunto, a fin de trazar un panorama futuro para el estudio de la TPB en el marketing ecológico.

\section{METODOLOGÍA}

Para alcanzar el objetivo de proporcionar una revisión sistémica de literatura que busque identificar, evaluar y resumir todos los estudios pertinentes, a través de un proceso replicable y transparente (Tranfield et al., 2003), se usó el protocolo sugerido por Cronin et al. (2008), que consistió en una metodología basada en una serie de búsquedas en bases de datos, con prioridades establecidas en la selección del material y la producción de una síntesis del campo al final (Creswell, 2010). A continuación, se presenta el paso a paso de la investigación realizada.

\section{Selección del tópico de repaso}

El primer paso fue considerar que el concepto de la teoría del comportamiento planificado en el marketing aparece asociado a fundamentos teóricos (por ejemplo, decisión de compra, intención de compra y actitudes). Por lo tanto, el campo de la revisión fue delimitado a solo la decisión de compra de productos orgánicos. Esto, sustentado con la regla general establecida por Cronin et al. (2008).

\section{Búsqueda de la literatura}

La búsqueda de literatura fue realizada principalmente en ScienceDirect, Taylor and Francis, Oxford University Press, Springer, Emerald Insight y SAGE Journals, donde se ingresaron diferentes términos de búsqueda y se ajustaron parámetros para acotar los resultados. Cabe señalar que la elección de las bases de datos se dio por ser hoy de las de literatura académica de mayor rigurosidad científica, las cuales indexan una amplia y variada gama de revistas de diferentes áreas del conocimiento y cuentan con gran aceptación entre los investigadores.

En el campo de búsqueda de las plataformas de datos, solo se adoptaron los descriptores TPB (theory of planning behavior) y organic products, visto que el objetivo fue definido sobre la teoría del comportamiento planificado en el marketing ecológico; con esto, se mitigaron posibles inconvenientes en la dispersión de resultados (Cronin et al., 2008). Las búsquedas fueron filtradas por título y resumen, en un recorte temporal establecido de las últimas tres décadas. Dicha opción está conectada con la intención de llenar una posible laguna existente desde la aparición de la TPB en 1985, sin generar hallazgos superpuestos.

\section{Recopilación de literatura, lectura y análisis}

Las búsquedas realizadas retornaron 146 publicaciones que se encuentran en las bases de datos de mayor rigurosidad científica que fueron mencionadas en la sección anterior. A estas se aplicaron los criterios de exclusión establecidos por Cronin et al., (2008) y quedaron seleccionados artículos científicos que han sido debidamente validados (Podsakoff et al., 2005). Por lo tanto, la investigación no se limitó a publicaciones periódicas con el mayor impacto en sus campos, sino que se incluyeron todos los artículos de revistas indexadas por ScienceDirect, Taylor and Francis, Oxford University Press, Springer, Emerald Insight y SAGE Journals que contenían la expresión "teoría del comportamiento planificado" AND “marketing” AND "producto orgánico”. Después de esta primera exclusión, la búsqueda arrojó 123 artículos científicos publicados en revistas, según los índices SJR-Scopus de niveles Q1, Q2, Q3 y Q4, que fueron recolectados en forma preliminar.

Posteriormente, se realizó un análisis para verificar si los estudios ya identificados eran apropiados para los propósitos de esta investigación. Fueron priorizados artículos que estuvieran escritos en inglés, español y portugués, y que se enfocaran en "productos orgánicos". Con este procedimiento, se excluyeron 55 artículos y se mantuvo para el estudio un total de 68 artículos. 
Finalmente, se completó el análisis de contenido al sistematizar los 68 artículos seleccionados, a través del software NVivo. Con esto, se determinó la homogeneidad y la correlación entre los artículos, al usar los indicadores de similitud basados en los coeficientes de correlación de Pearson. Se establecieron las distancias o similitudes entre los artículos consultados y procesados a través de una representación gráfica (dendograma). Para completar el estudio, se realizó un análisis individualizado e independiente de los artículos, para lo cual se tomaron como criterios de análisis el enfoque de investigación, método, muestra cuantitativa y/o cualitativa, técnicas de recolección y análisis de datos, resultados y conclusiones.

\section{RESULTADOS}

\section{Teoría del comportamiento planificado (TPB)}

Según la TPB, los comportamientos importantes de las personas son intencionales y, aunque las limitaciones externas y personales dificultan el actuar, el determinante inmediato del comportamiento es la intención de la persona de realizar ese comportamiento (Nuttavuthisit y Thøgersen, 2017). Por lo tanto, la TPB propone tres variables que influyen en las intenciones de comportamiento: la actitud, las normas subjetivas y el control percibido.

La actitud es la que se describe como una ruta psicosomática para evaluar una cosa exacta con amabilidad o descrédito (Eagly y Chaiken, 2007). Es decir, la actitud hacia el comportamiento se refiere al grado en que la persona tiene una evaluación favorable o desfavorable del comportamiento en cuestión (Ajzen y Driver, 1991). Las normas subjetivas actúan cuando personas importantes en el mundo piensan que el individuo debe involucrarse en un comportamiento específico y la inspiración del individuo cumple con el pensamiento de estas personas (Ajzen y Fishben, 1980). Es decir, el segundo predictor es un factor social que se refiere a la presión social percibida para realizar o no el comportamiento (Ajzen y Driver, 1991). Y el control percibido se relaciona con la percepción de un individuo sobre el grado de facilidad y las dificultades para realizar tal comportamiento (Achchuthan, Umanakenan y Kajenthiran, 2017).

Estas tres variables han permitido que la TPB se haya utilizado en numerosos estudios (ejemplo, Álvarez, López y González, 2015; Aertsens et al., 2011; Barber, 2012; Berenguer y Corraliza, 2000; Cherian y Jacob, 2012; Dunlap et al., 2000; Ertz y Sarigöllü, 2018; Hartmann y Apaolaza, 2012; Higuchi, 2015; Junior et al., 2014a; Neagu, 2012; Pires et al., 2014; Seman et al., 2019; Torres y Ayerbe, 2018; Trivedi et al., 2018; Yadav y Pathak, 2017) centrados en el comportamiento de compra en torno a lo ambiental.

\section{Panorama de las publicaciones}

Las revistas indexadas y sus respectivas clasificaciones se relacionan en la tabla 1. Se puede notar una concentración de publicaciones en revistas científicas con áreas de estudio enfocadas principalmente en Business, Management and Accounting; Agricultural and Biological Sciences, Economics and Finance, Environmental Science, Marketing, Psychology y Social Sciences. Así, el tema en revisión presenta una gran dispersión en las revistas que lo tratan, donde la revista Journal of Cleaner Production es la que mayor cuota de publicaciones posee, con un 10,29 \% del total, seguida por las revistas científicas Food Quality and Preference, Journal of Business Ethics, Journal of Retailing and Consumer Services, Appetite, International Journal of Sustainable Development \& World Ecology, British Food Journal y Journal of Social Issues, las cuales poseen el 22,05\% del total de publicaciones analizadas. En cuanto a las otras revistas consultadas, tuvieron una sola publicación relevante con la TPB. 


\section{Tabla 1}

Cantidad de publicaciones por revistas indexadas y su clasificación

\begin{tabular}{|c|c|c|}
\hline Clasificación & Revista & Cantidad \\
\hline \multirow{27}{*}{ Q1 } & Annual Review of Environment and Resources & 1 \\
\hline & Appetite & 2 \\
\hline & $\begin{array}{l}\text { Corporate Social Responsibility and } \\
\text { Environmental Management }\end{array}$ & \\
\hline & Ecological Economics & 1 \\
\hline & Environment and Behavior & 1 \\
\hline & ESIC Market & 1 \\
\hline & Estudios y Perspectivas en Turismo & 1 \\
\hline & European Journal of Marketing & 1 \\
\hline & Food Quality and Preference & 1 \\
\hline & $\begin{array}{l}\text { International Entrepreneurship and Management } \\
\text { Journal }\end{array}$ & 3 \\
\hline & $\begin{array}{l}\text { International Journal of Fashion Design, } \\
\text { Technology and Education }\end{array}$ & 1 \\
\hline & $\begin{array}{l}\text { International Journal of Sustainable Development } \\
\& \text { World Ecology }\end{array}$ & \\
\hline & Journal of Business Ethics & 1 \\
\hline & Journal of Business Research & \\
\hline & Journal of Cleaner Production & 2 \\
\hline & Journal of Consumer Marketing & 2 \\
\hline & Journal of Environmental Management & 1 \\
\hline & Journal of Marketing Communications & 7 \\
\hline & Journal of Retailing and Consumer Services & 1 \\
\hline & Journal of Social Issues & 1 \\
\hline & Leisure Sciences & 1 \\
\hline & Natural Hazards & 2 \\
\hline & Resources, Conservation and Recycling & 2 \\
\hline & Revista de Administração Mackenzie & 1 \\
\hline & Social and Personality Psychology Compass & 1 \\
\hline & Sustainable Cities and Society & 1 \\
\hline & Sustainable Production and Consumption & 1 \\
\hline \multirow{10}{*}{ Q2 } & British Food Journal & 2 \\
\hline & Journal of Applied Social Psychology, & 1 \\
\hline & Journal of Food Products Marketing & 1 \\
\hline & Marketing Intelligence \& Planning & 1 \\
\hline & Organic Agriculture & 1 \\
\hline & Psicothema & 1 \\
\hline & $\begin{array}{l}\text { Revista Española de Investigaciones } \\
\text { Sociológicas }\end{array}$ & 1 \\
\hline & Service Industries Journal & 1 \\
\hline & Spanish Journal of Agricultural Research & 1 \\
\hline & $\begin{array}{l}\text { The International Review of Retail, Distribution } \\
\text { and Consumer Research }\end{array}$ & \\
\hline
\end{tabular}




\begin{tabular}{|c|c|c|}
\hline \multirow{7}{*}{ Q3 } & African Journal of Business Management & 1 \\
\hline & Asian Social Science & 1 \\
\hline & Economic Research-Ekonomska Istraživanja & 1 \\
\hline & Environmental Practice & 1 \\
\hline & $\begin{array}{l}\text { International Journal of Hospitality \& Tourism } \\
\text { Administration }\end{array}$ & \\
\hline & Suma de Negocios & 1 \\
\hline & Wulfenia & 1 \\
\hline \multirow{11}{*}{ Q4 } & Apuntes & 1 \\
\hline & Economic Insights-Trends \& Challenges & 1 \\
\hline & $\begin{array}{l}\text { International Journal of Business and Social } \\
\text { Science }\end{array}$ & 1 \\
\hline & Journal of Business Studies & 1 \\
\hline & Journal of Global Scholars of Marketing Science & 1 \\
\hline & Psicologia em Estudo & 1 \\
\hline & Revista Innovar & 1 \\
\hline & Romanian economic journal & 1 \\
\hline & Social Sciences & 1 \\
\hline & $\begin{array}{l}\text { Studia Universitatis Vasile Goldis Arad, Seria } \\
\text { Stiintele Vietii }\end{array}$ & 1 \\
\hline & & 1 \\
\hline
\end{tabular}

Fuente: elaborado a partir de los cuartiles de Scimago Journal y Country Rank (2019).

Ahora bien, el número de publicaciones sobre la TPB en la decisión de compra de productos ecológicos demuestra que el desarrollo del tema en el área de la administración, el medio ambiente, la psicología y las ciencias sociales tiende a aumentar en las principales revistas científicas internacionales (figura 1). A partir de 2010, se evidencia un crecimiento constante en el número de publicaciones realizadas por año, hasta llegar a 2017 y 2018 , cuando se realizan diez y nueve publicaciones respectivamente. Y 2019 ya contaba con ocho publicaciones y posibilidades de aumento de ese número, si consideramos que la investigación fue concluida en noviembre de ese año. Esto evidencia la importancia del tema. 


\section{Figura 1}

Cantidad de publicaciones por año sobre temas relacionados con la teoría del comportamiento planificado, el medio ambiente y el marketing

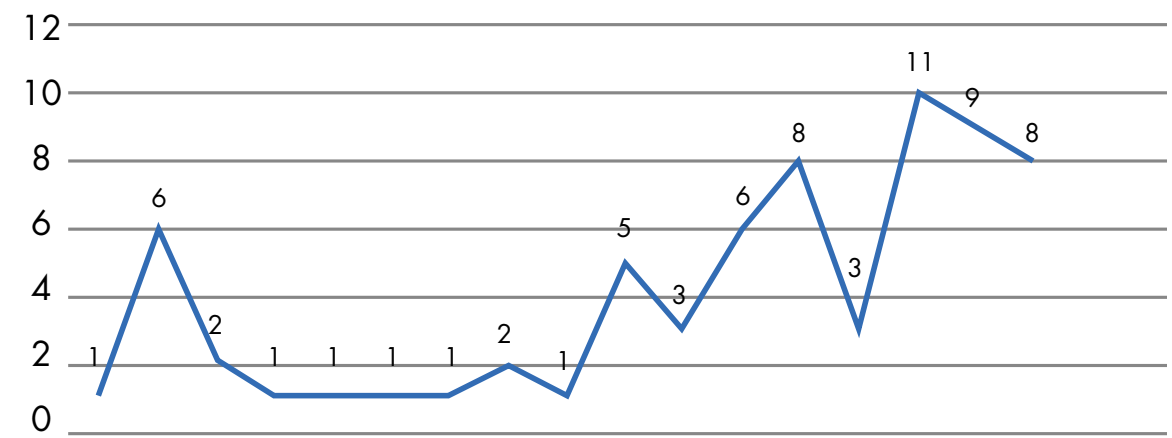

19912000200220032005200620072010201120122013201420152016201720182019

Fuente: Elaboración propia.

\section{Áreas de estudio}

Se realizó un análisis exploratorio del contenido de los diversos estudios analizados. La figura 2 presenta los resultados en una representación de las quinientas palabras con más de cuatro letras que se mencionaron con mayor frecuencia en estos artículos. Se destacan palabras como consumidor verde, marketing verde y comida orgánica, de acuerdo con el área de estudio, pero que están íntimamente relacionadas con otras palabras de nivel secundario, tales como comportamiento de compra, intención de compra y productos orgánicos, lo cual evidenció la preocupación en los estudios sobre productos orgánicos, marketing de productos orgánicos, TPB y consumidores verdes como principales temas de interés y de los cuales se extrajo información resumida sobre el tema a analizar en términos del tipo de estudio, tipo de análisis, conclusiones, alcance y similitudes entre los estudios.

\section{Figura 2}

Representación de las quinientas palabras más usadas en los artículos analizados

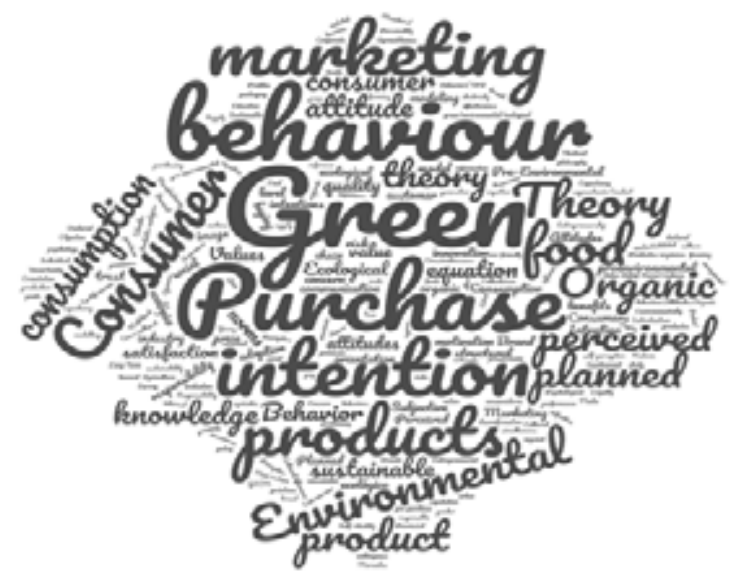

Fuente: elaboración propia. 
La figura 3 presenta los resultados de los artículos analizados en términos de la similitud de las palabras utilizadas en función del coeficiente de correlación de Pearson. Como se pudo observar, el análisis permite la identificación de varios grupos de artículos que resultan más similares entre sí cuando la figura se lee de abajo hacia arriba. De la misma manera, se entiende cómo hay claramente dos grupos principales y mutuamente distintos. El primer grupo de artículos (identificado en la parte superior de la figura), abarca sobre todo artículos que estudian el comportamiento ambiental, las dinámicas del marketing ecológico y sus diferentes predisposiciones. Mientras que el clúster que contiene el resto de los artículos, aunque de naturaleza más diversa, contiene sobre todo artículos que abordan la intención y el comportamiento de compra.

\section{Figura 3}

Agrupación de artículos por similitud de palabras

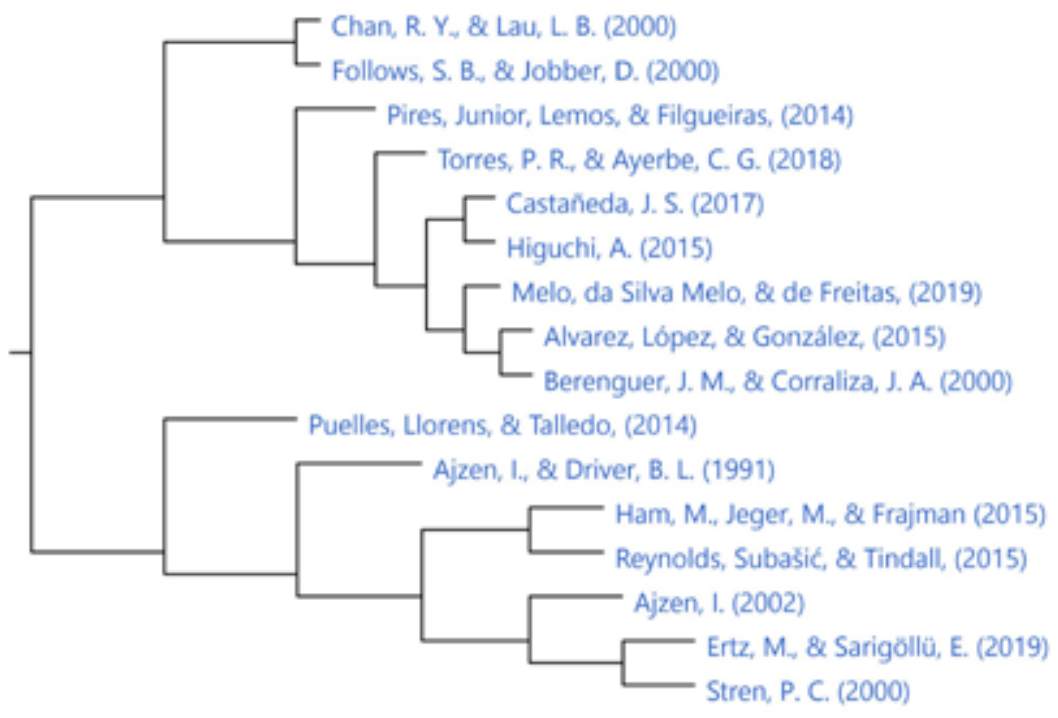




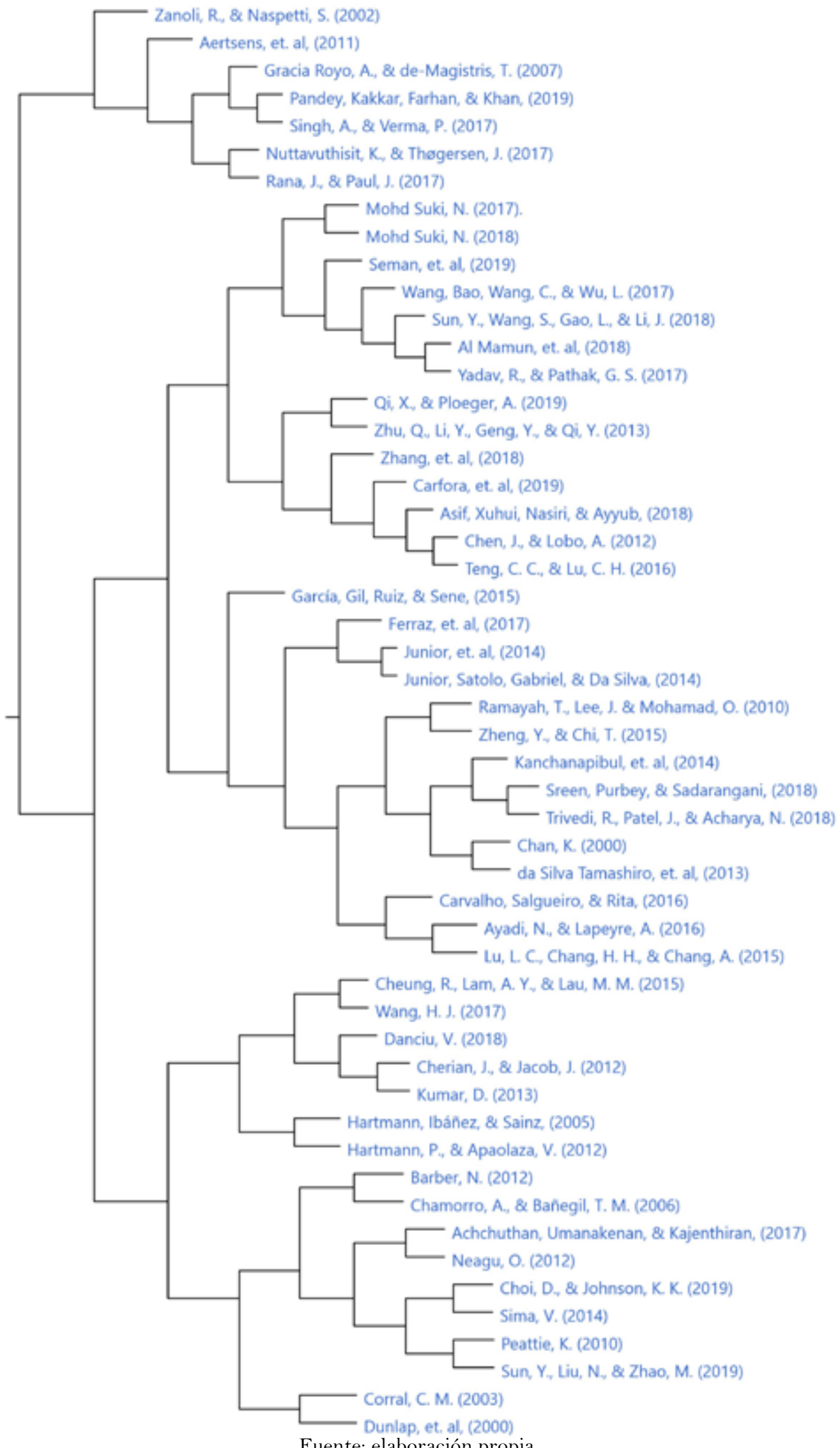


El análisis también confirma que la TPB se ha abordado en diversos campos relacionados con el medio ambiente, como se refleja en la distancia entre algunos de los artículos analizados. Por ejemplo, el artículo de Puelles-Gallo et al. (2014), centrado en la intención de compra hacia productos ecológicos de alimentación bajo la dualidad marca de fabricante versus marca de distribuidor; los artículos de Peattie (2010) y Sun et al. (2019), centrados en identificar, analizar y comprender al consumidor verde; o los artículos de Ayadi y Lapeyre (2016) y Chen y Lobo (2012), centrados en la disposición a pagar por productos orgánicos. Esta investigación también consideró los temas estudiados y sus respectivas conclusiones clave (tabla 2). Se puede apreciar que el mayor número de estudios se enfoca en el comportamiento de compra y en la intención de compra de productos orgánicos, donde la compra real depende de diversos factores del comportamiento, entre los cuales se destacan la actitud, el conocimiento y las creencias.

\section{Tabla 2}

\section{Temas de análisis}

\begin{tabular}{|c|c|c|}
\hline Tema de análisis & Principales conclusiones & Estudios \\
\hline Comportamiento & $\begin{array}{l}\text { - Las intenciones y comportamientos pueden } \\
\text { predecirse con precisión considerable a partir } \\
\text { de las actitudes hacia los comportamientos, las } \\
\text { normas subjetivas y las percepciones sobre el } \\
\text { control del comportamiento. } \\
\text { - La TPB es una teoría conductual adecuada para } \\
\text { explicar la actitud y el comportamiento del } \\
\text { consumidor en el mercado orgánico, incluso } \\
\text { cuando se incluyen motivos de consumo en el } \\
\text { análisis y pocas modificaciones estructurales con } \\
\text { respecto al modelo original. }\end{array}$ & $\begin{array}{l}\text { Ajzen (2002), Aizen y Driver (1991), } \\
\text { Corral (2003), Danciu (2018), Reynolds } \\
\text { et al. (2015) }\end{array}$ \\
\hline Comportamiento ambiental & $\begin{array}{l}\text { - La participación basada en el comportamiento } \\
\text { proambiental afecta las actitudes hacia este } \\
\text { comportamiento indirectamente. } \\
\text { - Existe una preocupación por el ambiente, donde } \\
\text { las personas consideran importantes los atributos } \\
\text { sociales y ambientales. } \\
\text { - Pocas contribuciones asociadas con promover } \\
\text { el comportamiento sostenible y el desarrollo } \\
\text { infantil, claves para incentivar cambios } \\
\text { sostenibles. } \\
\text { - La preocupación ambiental del consumidor } \\
\text { influye en la intención de compra. } \\
\text { - Si bien la preocupación por el medio ambiente } \\
\text { a nivel individual es alta, cuando las personas } \\
\text { evalúan la preocupación de los demás los } \\
\text { porcentajes bajan. }\end{array}$ & $\begin{array}{l}\text { Álvarez et al. (2015), Barber (2012), } \\
\text { Berenguer y Corraliza (2000), Cherian } \\
\text { y Jacob (2012), Dunlap et al. (2000), } \\
\text { Ertz y Sarigöllü (2018), Hartmann y } \\
\text { Apaolaza (2012), Hartmann, Ibañez y } \\
\text { Sainz (2005), Higuchi (2015), Junior et } \\
\text { al. (2014a), Neagu (2012), Pires et al. } \\
\text { (2014), Seman et al. (2019), Torres y } \\
\text { Ayerbe (2018), Trivedi et al. (2018) }\end{array}$ \\
\hline Comportamiento de compra & $\begin{array}{l}\text { - El comportamiento de compra de los sujetos es } \\
\text { precedido por la intención de compra, que es lo } \\
\text { que realmente importa en su decisión. } \\
\text { - La falta de información es una barrera para el } \\
\text { consumo de alimentos orgánicos. } \\
\text { - El comportamiento ambientalmente significativo } \\
\text { depende de una amplia gama de factores } \\
\text { causales, tanto generales como específicos } \\
\text { comportamentales. } \\
\text { - No incluir la variable intención contribuye } \\
\text { a la baja correlación entre las actitudes y el } \\
\text { comportamiento ambiental. }\end{array}$ & $\begin{array}{l}\text { Achchuthan et al. (2017), Aertsens et } \\
\text { al. (2011), Chamorro y Bañegil (2006), } \\
\text { Chan y Lau (2000), Cheung et al. } \\
\text { (2015), Choi y Johnson (2019), Carfora } \\
\text { et al. (2019), da-Silva et al. (2013), } \\
\text { Follows y Jobber (2000), Junior et al. } \\
\text { (2014b), Kanchanapibul et al. (2014), } \\
\text { Kumar (2013), Melo et al. (2019) }\end{array}$ \\
\hline
\end{tabular}


Comportamiento de compra
- Una actitud positiva hacia la compra y la creencia de que otras personas importantes compran estos productos es la predicción más directa y destacada de la intención de compra de productos ecológicos.

- Cuanto más conocedores son los consumidores sobre los problemas ambientales, más tienen la intención de comprar estos productos.

- En una alta conveniencia de compra, la intención de consumo de alimentos verdes tiende a generar comportamientos de consumo.

- La intención de comprar alimentos orgánicos y la confianza del consumidor en los productores e instituciones influye en la decisión de compra del consumidor de alimentos orgánicos.

- La decisión de compra de productos alimenticios orgánicos depende de la intención de compra y es un precursor del comportamiento de compra final. Además, la intención de compra depende de las actitudes y el conocimiento del producto orgánico.

- En alimentos orgánicos, las compras dependen de actitudes hacia la salud y beneficios ambientales proporcionados por lo orgánico, junto con el conocimiento de estos productos.

- Tanto el producto como las regulaciones influyen significativamente en la evaluación previa a la compra y esto se asocia con intenciones de compra a través del efecto mediador de creencias, actitudes y la evaluación previa.

- La "autocomplacencia" y la "búsqueda de variedades" influyen significativamente en las creencias y actitudes del consumidor hacia lo orgánico.

Intención de compra
- El entorno de consumo actual y las características de los consumidores influyen directamente en la intención de compra e incrementó la capacidad predictiva del modelo. - La actitud y la conciencia afectan directamente la intención de compra de alimentos orgánicos.

- La preocupación por el medio ambiente es un predictor no significativo de la intención de compra de alimentos orgánicos, donde los motivos egoístas, como la preocupación por la salud, son el mejor predictor de la intención de compra de alimentos orgánicos.

- Cuanto más altas son las intenciones de la persona, mayor es la expectativa de que lo intente y lo más probable es que logre realizar ese comportamiento.

- El efecto del individualismo, la actitud hacia los negocios y la propensión a la lealtad en cada dimensión de las creencias éticas influyen en la intención de compra del consumidor hacia los productos ecológicos.

- La intención de compra de los consumidores hacia lo orgánico está influenciada por presiones sociales percibidas y su disposición a ceder a la presión.

- La ambivalencia actitudinal puede desempeñar un rol en desarrollar la intención o incluso desempeñar un papel moderador en la relación actitud-comportamiento.
Mohd-Suki (2017), Peattie (2010), Rana

y Paul (2017), Sima (2014), Singh y Verma (2017), Stern (2000), Sun et al. (2019), Wang (2017), Zanoli y Naspetti (2002), Zhu et al. (2013)

Ayadi y Lapeyre (2016), Chen y Lobo (2012), Nuttavuthisit y Thøgersen (2017), Gracia y de-Magistris (2007)

Al-Mamun et al. (2018), Asif et al. (2018), Carvalho et al. (2016), Chan (2000), Batista Ferraz et al. (2017), García-Rodríguez et al. (2015), Ham et al. (2015), Lu et al., (2015), Mohd-Suki (2018), Pandey et al. (2019), Ramayah et al. (2010), Qi y Ploeger (2019), Sreen et al. (2018), Sun, et al. (2018), Teng y Lu (2016), Wang et al. (2017), Yadav y Pathak (2017), Zhang et al. (2018), Zheng y Chi (2015) 


\begin{tabular}{l|l|l}
\hline Dinámicas de mercadeo ambiental & $\begin{array}{l}\text { - La gente que consume productos ecológicos } \\
\text { lo hace por convicciones personales bastantes } \\
\text { fuertes sobre nutrición sana o cuidado del medio } \\
\text { ambiente. } \\
\text { - Los instrumentos de marketing pueden modificar } \\
\text { la intención de compra, aunque esta influencia } \\
\text { se ve más en los productos de marca del } \\
\text { fabricante. } \\
\begin{array}{l}\text { Las normas subjetivas en la intención de compra } \\
\text { de productos ecológicos no son determinantes } \\
\text { en personas con fuertes convicciones con } \\
\text { respecto a productos ecológicos. }\end{array}\end{array}$ & $\begin{array}{l}\text { Castañ } \\
(2014)\end{array}$ \\
\hline \\
Fuente: elaboración propia.
\end{tabular}

Fuente: elaboración propia.

Con respecto a los estudios centrados en el comportamiento, el comportamiento ambiental y la decisión de compra, se destacan las actitudes, las normas subjetivas y las percepciones sobre el control del comportamiento como variables adecuadas para explicar el comportamiento del consumidor en el mercado orgánico y se hallan líneas de investigación como las siguientes: a) estudios sobre la preocupación ambiental, b) estudios sobre el comportamiento sostenible, c) estudios sobre la salud y los beneficios ambientales proporcionados por los alimentos orgánicos, y d) estudios sobre la confianza del consumidor en los productores. Estas, a su vez, concluyen que las intenciones y los comportamientos pueden predecirse a partir de la TPB, donde debe considerarse la afectación de los atributos sociales y ambientales.

\section{Tipos de estudios}

Finalmente, se analizó la tipología de los estudios con respecto al tipo de análisis realizado. Como se establece en la tabla 3, 15 estudios son puramente teóricos, 53 son empíricos y, de ellos, 1 es cualitativo, donde se hace un análisis de contenidos, y 52 son cuantitativos, donde la mayoría aplica técnicas estadísticas y econométricas cuantitativas, tales como análisis de ecuaciones estructurales, modelos de regresión múltiple y análisis de mínimos cuadrados parciales. El análisis de la tabla 3 confirma que las metodologías cualitativas siguen siendo escasas en este campo, incluso si esto abre la comprensión de los significados de fenómenos en estudio (Patton, 2002).

\section{Tabla 3}

Tipo de estudio y tipo de análisis

\begin{tabular}{|c|c|c|c|}
\hline Tipo de estudio & & Tipo de análisis & Referencias \\
\hline Revisión teórica & & & $\begin{array}{l}\text { Achchuthan et al. (2017), Aizen y Driver } \\
\text { (1991), Aizen (2002), Castañeda (2017), } \\
\text { Chamorro y Bañegil (2006), Danciu } \\
\text { (2018), Dunlap et al. (2000), Higuchi } \\
\text { (2015), Neagu (2012), Peattie (2010), } \\
\text { Pires et al. (2014), Rana y Paul (2017), } \\
\text { Reynolds et al. (2015), Sima (2014), } \\
\text { Stern (2000) }\end{array}$ \\
\hline Estudios empíricos & cuantitativo & $\begin{array}{l}\text { Regresión múltiple- } \\
\text { análisis de varianza }\end{array}$ & $\begin{array}{l}\text { Aertsens et al. (2011), da-Silva et al. } \\
\text { (2013), Kumar (2013), Melo et al. } \\
\text { (2019), Nuttavuthisit y Thøgersen (2017), } \\
\text { Singh y Verma (2017), }\end{array}$ \\
\hline
\end{tabular}




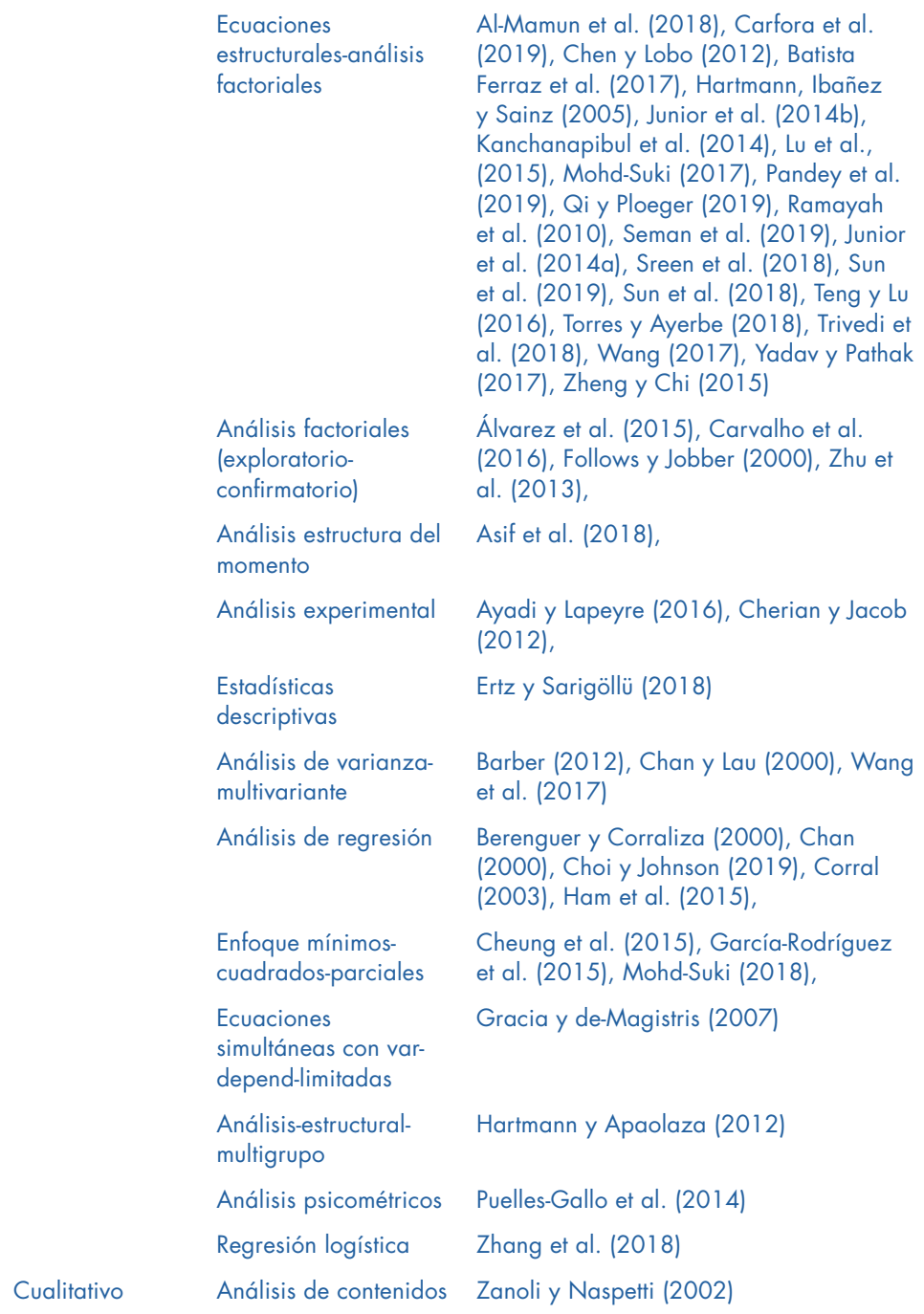

Fuente: elaboración propia.

\section{Implicaciones y futuras líneas de investigación}

A partir de los resultados presentados por los diferentes estudios, se puede identificar diversas implicaciones, tanto para la teoría como para la práctica. Una de las conclusiones alcanzadas por los estudios se refiere a que la TPB es una teoría conductual adecuada para explicar el comportamiento del consumidor, debido a que las intenciones y comportamientos pueden predecirse con una precisión considerable, a partir de las actitudes hacia los comportamientos, las normas subjetivas y las percepciones sobre el control del comportamiento (Ajzen, 2002; Ajzen y Driver, 1991; Corral, 2003); por lo tanto, las investigaciones aplicadas deben garantizar el abordaje de estos temas abiertamente. Por ejemplo, Pires et al. (2014) sostienen que existen pocas contribuciones asociadas a promover e incentivar el comportamiento sostenible. Esto apunta a la necesidad de analizar los comportamientos de los consumidores en diferentes ámbitos y en particular el ambiental que convoca esta investigación, donde se encuentran estudios como el de Torres y Ayerbe (2018), el cual concluye que el comportamiento proambiental sigue un proceso lógico donde aumentan gradualmente la diversidad y la intensidad de las acciones previstas a favor del medio ambiente. Por lo tanto, estudios como el de Junior et al. (2014a) se vuelven importantes al encontrar que los atributos sociales y ambientales son considerados importantes para las personas. Se entiende, por un lado, que la preocupación ambiental del consumidor influye en la intención de compra (Hartmann y Apaolaza, 2012). No 
obstante, esta preocupación por el medio ambiente no es un predictor significativo de la intención de compra, donde los motivos egoístas, como la preocupación por la salud, son mejores predictores (Asif et al., 2018). Y, por otro, si bien la preocupación por el medio ambiente a nivel individual es alta, cuando las personas evalúan la preocupación de los demás, los porcentajes bajan considerablemente (Berenguer y Corraliza, 2000).

Otra conclusión con importantes implicaciones en el comportamiento de compra es que dicho comportamiento es precedido por la intención de comprar y lo que realmente importa en su decisión de esa compra (Junior et al., 2014b; Zhu et al., 2013). Por tanto, esta intención es afectada directamente por una actitud positiva hacia la compra y la creencia de que personas importantes compran (Choi y Johnson, 2019). Ahora bien, no incluir la variable intención contribuye a la baja correlación entre las actitudes y el comportamiento ambiental (Follows y Jobber, 2000). Sin embargo, se debe dejar claro que el comportamiento ambientalmente significativo depende de una amplia gama de factores causales, tanto generales como específicos, del comportamiento (Stern, 2000). Estos hallazgos resaltan que las creencias y el conocimiento por un producto en particular afectan las actitudes que finalmente influyen en las intenciones de compra (Nuttavuthisit y Thøgersen, 2017; Gracia y de-Magistris, 2007). Es decir, las actitudes y creencias de los consumidores están influenciadas positiva y directamente por el producto, la regulación y el estilo de vida, lo cual se traduce en un comportamiento de compra (Chen y Lobo, 2012).

Por otro lado, los resultados también demuestran cómo el efecto del individualismo, la actitud hacia los negocios y la propensión a la lealtad en cada dimensión de las creencias éticas influyen en la intención de compra del consumidor hacia los productos ecológicos (Lu et al., 2015). Es decir, la intención de compra de los consumidores hacia lo ecológico está influenciada por presiones sociales percibidas y su disposición a ceder a la presión (Zheng y Chi, 2015).

En síntesis, se puede decir que la mayoría de los estudios se centran en comprender los aspectos relacionados con el comportamiento del consumidor ecológico, sobre todo en temas asociados con lo orgánico, lo ambiental y lo verde. Por lo anterior, se necesitan modelos desagregados de la TPB para analizar el comportamiento y la intención de compra de los consumidores ecológicos. Sin embargo, se necesitan más estudios para confirmar los resultados encontrados y también para analizar los modelos del comportamiento desarrollados en el marketing ecológico.

Es importante mencionar que, en la mayoría de los artículos relacionados con productos orgánicos (Asif et al., 2018; Pandey et al., 2019; Qi y Ploeger, 2019; Zhang et al., 2018), se incluye la variable conciencia frente a la salud, que se encuentra relacionada con los aportes que realizan estos productos a mantener hábitos de vida saludables. Tendencia marcada en el consumidor actual, quien busca que los productos consumidos y utilizados reduzcan los impactos negativos generados en las personas y en el medio ambiente.

De la misma manera, es importante analizar cómo otras variables no consideradas en los artículos analizados modifican la intención de compra del consumidor habitual de estos productos. En estos modelos podría incluirse la variable precio, como un influenciador importante en la intención de compra del consumidor, si se tiene en cuenta que algunos productos ecológicos y orgánicos necesitan procesos de producción complejos que incrementan los costos frente a los tradicionales; estos costos se trasmiten al consumidor final e incrementan el precio. Es común que consumidores con ingresos bajos sean muy susceptibles al precio (Feraz et al., 2017; Rana y Paul, 2017; Zhang et al., 2018). En algunas otras publicaciones (Achchuthan et al., 2017; Teng y Lu, 2016; Wang, 2017; Wang et al., 2017), se menciona la importancia del valor percibido de los clientes frente a la intención de compra de estos productos; debe tenerse en cuenta que esta variable es muy amplia, lo cual implica que son influenciados por la calidad, los riesgos, la publicidad y otros, que también pueden modificar la intención de compra. Por lo tanto, sería valioso tener un modelo integral que incorpore y valide diferentes variables y tenga en cuenta su prioridad para los clientes y cómo influyen en la intención de compra.

En consecuencia, se hace necesario constituir futuras líneas de investigación que busquen comprender mejor cómo se lleva a cabo el proceso de decisión de compra de un producto orgánico por parte de los consumidores y, con este fin, recurrir a teorías del comportamiento basadas en la TPB para comprender mejor las diferentes variables que afectan este proceso. Finalmente, los resultados demuestran cómo la mayoría de los estudios adoptaron enfoques cuantitativos y, por lo tanto, indican la necesidad de enfoques más cualitativos para comprender los comportamientos e intenciones de compra de productos ecológicos por parte de los consumidores. 


\section{CONCLUSIÓN}

Es evidente que las tendencias del consumidor cambian rápidamente y generan un reto importante para las empresas, que deben satisfacer sus gustos, deseos y requerimientos. La preocupación por el medio ambiente y sobre la salud de las personas es una tendencia del marketing que obliga a estructurar diferentes estrategias que permitan asegurar a los clientes que los productos y servicios no generen impactos ambientales negativos y preserven el entorno para futuras generaciones. Para predecir las intenciones de compra de estos consumidores frente a este reto, es utilizada frecuentemente la TPB, que incluye la actitud, las normas subjetivas y el control de comportamiento percibido. Aunque la mayoría de los estudios toman como base esta teoría, cada artículo adiciona una variable que amplía este modelo, con la finalidad de entender la intención de compra en el consumidor, y agregan variables como la conciencia ambiental o de salud, el valor y riesgos percibidos, y el precio, entre otras que pueden influenciar la decisión final.

Los estudios relacionados con la intención de compra de alimentos orgánicos muestran que la conciencia es un factor importante para los consumidores, ya que esta variable considera tanto la conciencia sobre el medio ambiente como la salud. Los alimentos orgánicos deben garantizar que sus características de producción y distribución utilicen materiales amigables y, a la vez, reduzcan los riesgos para la salud por el uso de sustancias nocivas. Además, se menciona que las normas subjetivas influyen en la intención de compra, en relación con las expectativas que otras personas tienen frente al comportamiento. Es importante destacar, entre las variables que no forman parte del modelo teórico, el uso del precio, que influye positivamente en personas de altos ingresos, quienes esperan que estos productos tengan alta calidad, e influyen negativamente en las personas de bajos ingresos, quienes esperan maximizar los beneficios recibidos por productos comprados. Estos estudios se aplican principalmente a países desarrollados, pocos en América Latina, por lo que sería interesante reconocer aquellas variables que refuerzan el TPB de la cultura latina y contrastan con las identificadas en los países desarrollados. 


\section{REFERENCIAS}

Achchuthan, S., Umanakenan, R., y Kajenthiran, K. (2017). Comprehensive model for understanding and enhancing pro-environmental purchase behavior: Towards a conceptual framework. Journal of Business Studies, 4(1).

Aertsens, J., Mondelaers, K., Verbeke, W., Buysse, J., y van-Huylenbroeck, G. (2011). The influence of subjective and objective knowledge on attitude, motivations and consumption of organic food. British food journal, 113(11), 1353-1378. https://doi.org/10.1108/00070701111179988

Ajzen, I. (2002). Perceived behavioral control, self-efficacy, locus of control, and the theory of planned behavior 1. Journal of Applied Social Psychology, 32(4), 665-683. https://doi.org/10.1111/j.1559-1816.2002.tbo0236.x

Ajzen, I. y Fishbein, M. (1980). Understanding Attitudes and Predicting Social Behavior. Prentice-Hall.

Ajzen, I., y Driver, B. (1991). Prediction of leisure participation from behavioral, normative, and control beliefs: An application of the theory of planned behavior. Leisuresciences, 13(3), 185-204. https://doi. org/10.1080/01490409109513137

Al-Mamun, A., Mohamad, M. R., Yaacob, M. R., y Mohiuddin, M. (2018). Intention and behavior towards green consumption among low-income households. Journal of Environmental Management, 227, 73-86. https://doi. org/10.1016/j.jenvman.2018.08.061

Álvarez, P., M. J. López, y González, E. (2015). El perfil del consumidor ecológico en España. Esic Market Economics and Business Journal, 46, 269-297. https://doi.org/10.7200/esicm.151.0462.1e

Asif, M., Xuhui, W., Nasiri, A., y Ayyub, S. (2018). Determinant factors influencing organic food purchase intention and the moderating role of awareness: A comparative analysis. Food Quality and Preference, 63, 144-150. https://doi. org/10.1016/j.foodqual.2017.08.006

Ayadi, N., y Lapeyre, A. (2016). Consumer purchase intentions for green products: Mediating role of WTP and moderating effects of framing. Journal of Marketing Communications, 22(4), 367-384. https://doi.org/10.1080/1 3527266.2014.888574

Barber, N. (2012). Consumers' intention to purchase environmentally friendly wines: a segmentation approach. International Journal of Hospitality \& Tourism Administration, 13(1), 26-47. https://doi.org/10.1080/15256480 .2012 .640183

Batista Ferraz, S., Buhamra, C., Laroche, M. y Rodríguez Veloso, A. (2017). Green products: A cross-cultural study of attitude, intention and purchase behavior. RAM. Revista de Administração Mackenzie, 18(5), 12-38. https://doi. org/10.1590/1678-69712017/administracao.v18n5p12-38

Berenguer, J., y Corraliza, J. (2000). Preocupación ambiental y comportamientos ecológicos. Psicothema, 12(3), 325-329.

Carfora, V., Cavallo, C., Caso, D., Del-Giudice, T., De-Devitiis, B., Viscecchia, R., Nardone, G., y Cicia, G. (2019). Explaining consumer purchase behavior for organic milk: Including trust and green self-identity within the theory of planned behavior. Food Quality and Preference, 76, 1-9. https://doi.org/10.1016/j.foodqual.2019.03.006

Carvalho, B., Salgueiro, M., y Rita, P. (2016). Accessibility and trust: the two dimensions of consumers' perception on sustainable purchase intention. International Journal of Sustainable Development E World Ecology, 23(2), 203209. https://doi.org/10.1080/13504509.2015.1110210

Castañeda, J. (2017). Mercado de productos agrícolas ecológicos en Colombia. Suma de Negocios, 8(18), 156-163. https://doi.org/10.1016/j.sumneg.2017.10.001

Chamorro, A., y Bañegil, T. (2006). Green marketing philosophy: a study of Spanish firms with ecolabels. Corporate Social Responsibility and Environmental Management, 13(1), 11-24. https://doi.org/10.1002/csr.83

Chan, K. (2000). Environmental consideration in purchase decisions of Hong Kong consumers. Environmental Practice, 2(1), 15-22. https://doi.org/10.1017/S1466046600000983

Chan, R., y Lau, L. (2000). Antecedents of green purchases: a survey in China. Journal of consumer marketing, 17(4), 338-357. https://doi.org/10.1108/07363760010335358 
Chen, J., y Lobo, A. (2012). Organic food products in China: determinants of consumers' purchase intentions. The International Review of Retail, Distribution and Consumer Research, 22(3), 293-314. https://doi.org/10.1080/0 9593969.2012.682596

Cherian, J., y Jacob, J. (2012). Green marketing: A study of consumers' attitude towards environment friendly products. Asian Social Science, 8(12), 117. http://dx.doi.org/10.5539/ass.v8n12p117

Cheung, R., Lam, A., y Lau, M. (2015). Drivers of green product adoption: the role of green perceived value, green trust and perceived quality. Journal of Global Scholars of Marketing Science, 25(3), 232-245.-https://doi.org/10.1 080/21639159.2015.1041781

Choi, D., y Johnson, K. (2019). Influences of environmental and hedonic motivations on intention to purchase green products: An extension of the theory of planned behavior. Sustainable Production and Consumption, 18, 145-155. https://doi.org/10.1016/j.spc.2019.02.001

Coddington, W. (1993). Environmental marketing: positive strategies for reaching the green consumer. McGraw-Hill Companies.

Corral, C. (2003). Sustainable production and consumption systems-cooperation for change: assessing and simulating the willingness of the firm to adopt/develop cleaner technologies. The case of the In-Bond industry in northern Mexico. Journal of Cleaner Production, 11(4), 411-426. https://doi.org/10.1016/So959-6526(02)00063-X

Creswell, J. (2010). Mapping the developing landscape of mixed methods research. SAGE Handbook of Mixed Methods in Social \& Behavioral Research, 2, 45-68. https://dx.doi.org/10.4135/9781506335193.n2

Cronin, P., Ryan, F., y Coughlan, M. (2008). Undertaking a literature review: a step-by-step approach. British Journal of Nursing, 17(1), 38-43. https://doi.org/10.12968/bjon.2008.17.1.28059

Da-Silva, T., da-Silveira, J. A., Merlo, E., y Ghisi, M. (2013). The relationship between ecological knowledge, ecological concern, ecological affection, subjective norms and the green purchase behavior in Brazil. African Journal of Business Management, 7(34), 3297-3314. https://doi.org/10.5897/AJBM12.1270

Danciu, V. (2018). The Changing Focus of Green Marketing: From Ecological to Sustainable Marketing (III). Romanian Economic Journal, 21(68), 121-144.

Dunlap, R. E. V. L., Van-Liere, K. D., Mertig, A., y Jones, R. E. (2000). New trends in measuring environmental attitudes: measuring endorsement of the new ecological paradigm: a revised NEP scale. Journal of Social Issues, 56(3), 425-442. https://doi.org/10.1111/0022-4537.00176

Eagly, A., y Chaiken, S. (2007). The advantages of an inclusive definition of attitude. Social Cognition, 25(5), 582-602. https://doi.org/10.1521/soco.2007.25.5.582

Elkington, J., y Hailes, J. (1989). The Green Consumer's Supermarket Shopping Guide: Shelf by Shelf Recommendations for Products which Don't Cost the Earth. Victor Gollancz.

Ertz, M., y Sarigöllü, E. (2018). The behavior-attitude relationship and satisfaction in proenvironmental behavior. Environment and Behavior, 51(9-10), 1106-1132. https://doi.org/10.1177/0013916518783241

Ferraz, S. B., Buhamra, C., Laroche, M., \& Veloso, A. R. (2017). Green products: A cross-cultural study of attitude, intention and purchase behavior. RAM. Revista de Administração Mackenzie, 18(5), 12-38.

Fisk, G. (1974). Marketing and the Ecological Crisis. Harper \& Row.

Follows, S., y Jobber, D. (2000). Environmentally responsible purchase behaviour: a test of a consumer model. European Journal of Marketing, 34(5/6), 723-746. https://doi.org/10.1108/03090560010322009

Fuller, D. (1999). Sustainable marketing: Managerial-ecological issues. Sage Publications.

Fuller, D., y Butler, D. (2015). Eco-marketing: a waste management perspective. En E. J. Wilson y W. C. Black (Eds.), Proceedings of the 1994 Academy of Marketing Science (AMS) Annual Conference (pp. 331-331). Springer. https:// doi.org/10.1007/978-3-319-13162-7_89

Galindo, A. (2001). Psicología del consumidor mexicano. Revista Segmento. ITAM, noviembre, (14). 
García-Rodríguez, F., Gil-Soto, E., Ruiz-Rosa, I., y Sene, P. (2015). Entrepreneurial intentions in diverse development contexts: A cross-cultural comparison between Senegal and Spain. International Entrepreneurship and Management Journal, 11(3), 511-527. https://doi.org/10.1007/s11365-013-0291-2

Gracia-Royo, A., y de-Magistris, T. (2007). Organic food product purchase behaviour: a pilot study for urban consumers in the South of Italy. Spanish Journal of Agricultural Research, 5(4), 439-451.

Ham, M., Jeger, M., y Frajman, A. (2015). The role of subjective norms in forming the intention to purchase green food. Economic Research-Ekonoms Kaistrazivanja, 28(1), 738-748. https://doi.org/10.1080/1331677X.2015.1083875

Hartmann, P., Ibáñez, V. y Sainz, F. (2005). Green branding effects on attitude: functional versus emotional positioning strategies. Marketing Intelligence \& Planning. https://doi.org/10.1108/02634500510577447

Hartmann, P., y Apaolaza, V. (2012). Consumer attitude and purchase intention toward green energy brands: The roles of psychological benefits and environmental concern. Journal of Business Research, 65(9), 1254-1263. https://doi. org/10.1016/j.jbusres.2011.11.001

Henion, K. y Kinnear, T. (1976). A guide to ecological marketing. En K. Henion y T. Kinnear (Eds.), Ecological Marketing. American Marketing Association.

Higuchi, A. (2015). Características de los consumidores de productos orgánicos y expansión de su oferta en Lima. Apuntes, 42(77), 57-89.

Junior, S., da-Silva, D., Satolo, E., Magalhães, M. Putti, F., y de-Oliveira, W. (2014a). Environmental concern has to do with the stated purchase behavior of green products at retail? Social Sciences, 3(1), 23-30. https://doi. org/10.11648/j.ss.20140301.15

Junior, S., Satolo, E., da-Silva Gabriel, M. L. y da-Silva, D. (2014b). The relationship between environmental concern and declared retail purchase of green products. International Journal of Business and Social Science, 5(2).

Kanchanapibul, Lacka, Wang, y Chan, (2014). An empirical investigation of green purchase behaviour among the young generation. Journal of Cleaner Production, 66, 528-536. https://doi.org/10.1016/j.jclepro.2013.10.062

Kassarjian, H. (1971). Incorporating ecology into marketing strategy: The case of air pollution. Journal of Marketing, 35(3), 61-65. https://doi.org/10.1177/002224297103500310

Kumar, D. (2013). Does Green Marketing works with consumers? Wulfenia Journal, 20(3), 329-347.

Lu, L., Chang, H., y Chang, A. (2015). Consumer personality and green buying intention: The mediate role of consumer ethical beliefs. Journal of Business Ethics, 127(1), 205-219. https://doi.org/10.1007/s10551-013-2024-4

Melo, F., da-Silva, S., y de-Freitas, A. (2019). La relación entre conciencia ecológica y predisposición a pagar más en destinos turísticos que adoptan prácticas de sustentabilidad: un estudio con consumidores de Pernambuco-Brasil. Estudios y Perspectivas en Turismo, 28(2), 393-407.

Mohd-Suki, N. (2017). Green products usage: structural relationships on customer satisfaction and loyalty. International Journal of Sustainable Development E World Ecology, 24(1), 88-95. https://doi.org/10.1080/13504509.2016.116 9563

Mohd-Suki, N. (2018). Determinants of consumers' purchase intentions of organic vegetables: Some insights from Malaysia. Journal of Food Products Marketing, 24(4), 392-412. https://doi.org/10.1080/10454446.2017.1280717

Mohr, L., Webb, D., y Harris, K. (2001). Do consumers expect companies to be socially responsible? The impact of corporate social responsibility on buying behavior. Journal of Consumer Affairs, 35(1), 45-72. https://doi. org/10.1111/j.1745-6606.2001.tb00102.x

Neagu, O. (2012). Communication in the ecological marketing. Studia Universitatis "Vasile Goldis", Seria Stiintele Vietii (Life Sciences Series), 22(4).

Newholm, T., y Shaw, D. (2007). Studying the ethical consumer: A review of research. Journal of Consumer Behaviour: an international research review, 6(5), 253-270. https://doi.org/10.1002/cb.225

Nuttavuthisit, K., y Thøgersen, J. (2017). The importance of consumer trust for the emergence of a market for green products: The case of organic food. Journal of Business Ethics, 140(2), 323-337. https://doi.org/10.1007/s 10551015-2690-5 
Oliveira-Brochado, F., Oliveira-Brochado, A., y Caldeira, T. (2015). Os determinantes psicológicos do consumidor verde. Tourism \& Management Studies, 11(2), 104-111. http://dx.doi.org/10.18089/tms.2015.11213

Ottman, J. (1992). Industry's response to green consumerism. Journal of Business Strategy, 13(4), 3-7.

Pandey, Kakkar, Farhan, y Khan, (2019). Factors influencing organic foods purchase intention of Indian customers. Organic Agriculture, 1-8. https://doi.org/10.1007/s13165-018-0240-Z

Patton, M. Q. (2002). Two decades of developments in qualitative inquiry: A personal, experiential perspective. Qualitative social work, 1(3), 261-283.

Peattie, K. (2010). Green consumption: behavior and norms. Annual review of environment and resources, 35, 195-228. https://doi.org/10.1146/annurev-environ-032609-094328

Peattie, K., y Ratnayaka, M. (1992). Responding to the green movement. Industrial Marketing Management, 21(2), 103110. https://doi.org/10.1016/0019-8501(92)90004-D

Pires, P., Ribas Junior, R., Lemos, D., y Filgueiras, A. (2014). Ecocentrismo e comportamento: revisão da literatura em valores ambientais. Psicologia em Estudo, 19(4), 611-620. http://dx.doi.org/10.1590/1413-73722201204

Pita-Morales, L. A. (2016). Línea de tiempo: educación ambiental en Colombia. Praxis, 12(1), 118-125. https://doi. org/10.21676/23897856.1853

Podsakoff, P. M., MacKenzie, S. B., Bachrach, D. G., y Podsakoff, N. P. (2005). The influence of management journals in the 1980s and 1990s. Strategic Management Journal, 26(5), 473-488. https://doi.org/10.1002/smj.454

Polanski, M., y Anderson, N. (2006). A list of candidate cancer biomarkers for targeted proteomics. Biomarker Insights, 1. https://doi.org/10.1177/117727190600100001

Prothero, A. (1990). Green consumerism and the societal marketing concept: marketing strategies for the 1990's. Journal of Marketing Management, 6(2), 87-103. https://doi.org/10.1080/0267257X.1990.9964119

Puelles-Gallo, M., Llorens-Marín, M., y Talledo-Flores, H. (2014). El factor de la percepción de control como determinante en la intención de compra de productos ecológicos. Innovar, 24(54), 139-152. http://dx.doi. org/10.15446/innovar.v24n54.46655

Qi, X., y Ploeger, A. (2019). Explaining consumers' intentions towards purchasing green food in Qingdao, China: The amendment and extension of the theory of planned behavior. Appetite, 133, 414-422. https://doi.org/10.1016/j. appet.2018.12.004

Raiteri, M. (2015). El comportamiento del consumidor actual [Tesina de grado]. Universidad Nacional de Cuyo.

Ramayah, T., Lee, J., y Mohamad, O. (2010). Green product purchase intention: Some insights from a developing country. Resources, Conservation and Recycling, 54(12), 1419-1427. https://doi.org/10.1016/j.resconrec.2010.06.007

Rana, J., y Paul, J. (2017). Consumer behavior and purchase intention for organic food: A review and research agenda. Journal of Retailing and Consumer Services, 38, 157-165. https://doi.org/10.1 108/07363761211259223

Reynolds, K., Subašic, E., y Tindall, K. (2015). The problem of behaviour change: From social norms to an ingroup focus. Social and Personality Psychology Compass, 9(1), 45-56. https://doi.org/10.1111/spc3.12155

Sánchez-Villegas, (2014). Bioestadística amigable. En M. Á. Martínez-González, y F. J. Faulín (Eds.). Elsevier.

Scimago Journal y Country Rank (2019). https://www.scimagojr.com/aboutus.php

Seman, N., Govindan, K., Mardani, A., Zakuan, N., Saman, M., Hooker, R., y Ozkul, S. (2019). The mediating effect of green innovation on the relationship between green supply chain management and environmental performance. Journal of Cleaner Production, 229, 115-127. https://doi.org/10.1016/j.jclepro.2019.03.211

Shaw, D., y Shiu, E. (2002). An assessment of ethical obligation and self-identity in ethical consumer decision-making: a structural equation modelling approach. International Journal of Consumer Studies, 26(4), 286-293. https://doi. org/10.1046/j.1470-6431.2002.00255.x

Sima, V. (2014). Green Behaviour of the Romanian Consumers. Economic Insights-Trends \& Challenges, 66(3).

Singh, A., y Verma, P. (2017). Factors influencing Indian consumers' actual buying behaviour towards organic food products. Journal of Cleaner Production, 167, 473-483. https://doi.org/10.1016/j.jclepro.2017.08.106 
Sreen, N., Purbey, S., y Sadarangani, P. (2018). Impact of culture, behavior and gender on green purchase intention. Journal of Retailing and Consumer Services, 41, 177-189. https://doi.org/10.1016/j.jretconser.2017.12.002

Stern, P. (2000). Toward a coherent theory of environmentally significant behaviour. Journal of Social Issues, 56(3), 407-424.

Sun, Y., Liu, N., y Zhao, M. (2019). Factors and mechanisms affecting green consumption in China: A multilevel analysis. Journal of Cleaner Production, 209, 481-493. https://doi.org/10.1016/j.jclepro.2018.10.241

Sun, Y., Wang, S., Gao, L. y Li, J. (2018). Unearthing the effects of personality traits on consumer's attitude and intention to buy green products. Natural Hazards, 93(1), 299-314. https://doi.org/10.1007/s1 1069-018-3301-4

Teng, C., y Lu, C. (2016). Organic food consumption in Taiwan: Motives, involvement, and purchase intention under the moderating role of uncertainty. Appetite, 105, 95-105. https://doi.org/10.1016/j.appet.2016.05.006

Torres, P., y Ayerbe, C. (2018). Desarrollo del comportamiento proambiental en los individuos y sus determinantes. Reis: Revista Española de Investigaciones Sociológicas, 163, 59-78. https://doi.org/10.2307/26633303

Tranfield, D., Denyer, D., y Smart, P. (2003). Towards a methodology for developing evidence-informed management knowledge by means of systematic review. British Journal of Management, 14(3), 207-222. https://doi. org/10.1111/1467-8551.00375

Trivedi, R., Patel, J., y Acharya, N. (2018). Causality analysis of media influence on environmental attitude, intention and behaviors leading to green purchasing. Journal of Cleaner Production, 196, 11-22. https://doi.org/10.1016/j. jclepro.2018.06.024

Van-Dam, Y., y Apeldoorn, P. A. C. (1996). Sustainable marketing. Journal of Macromarketing, 16(2), 45-56. https://doi. org/10.1177/027614679601600204

Wang, H. (2017). Determinants of consumers' purchase behaviour towards green brands. The Service Industries Journal, 37(13-14), 896-918. https://doi.org/10.1080/02642069.2017.1365140

Wang, J., Bao, J., Wang, C., y Wu, L. (2017). The impact of different emotional appeals on the purchase intention for green products: The moderating effects of green involvement and Confucian cultures. Sustainable Cities and Society, 34, 32-42. https://doi.org/10.1016/j.scs.2017.06.001

Wang, R., y Strong, D. (1996). Beyond accuracy: What data quality means to data consumers. Journal of Management Information Systems, 12(4), 5-33. https://doi.org/10.1080/0742 1222.1996.11518099

Yadav, R., y Pathak, G. (2017). Determinants of consumers' green purchase behavior in a developing nation: Applying and extending the theory of planned behavior. Ecological Economics, 134, 114-122. https://doi.org/10.1016/j. ecolecon.2016.12.019

Zanoli, R., y Naspetti, S. (2002). Consumer motivations in the purchase of organic food: a means-end approach. British Food Journal, 104(8), 643-653. https://doi.org/10.1108/00070700210425930

Zhang, B., Fu, Z., Huang, J., Wang, J., Xu, S., y Zhang, L. (2018). Consumers' perceptions, purchase intention, and willingness to pay a premium price for safe vegetables: A case study of Beijing, China. Journal of Cleaner Production, 197, 1498-1507. https://doi.org/10.1016/j.jclepro.2018.06.273

Zheng, Y., y Chi, T. (2015). Factors influencing purchase intention towards environmentally friendly apparel: an empirical study of US consumers. International Journal of Fashion Design, Technology and Education, 8(2), 68-77. https://doi.org/10.1080/17543266.2014.990059

Zhu, Q., Li, Y., Geng, Y., y Qi, Y. (2013). Green food consumption intention, behaviors and influencing factors among Chinese consumers. Food Quality and Preference, 28(1), 279-286. https://doi.org/10.1016/j.foodqual.2012.10.005 